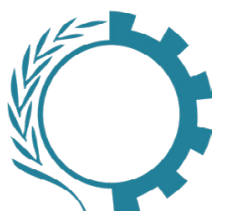

\title{
Effects of Hydrogen Peroxide on the Respiratory System of Dairy Industry Workers
}

Jamshid Rahimi, Assistant Professor, Department of Occupational Health Engineering, Research Center for Health, Safety and Environment (RCHSE), Alborz University of Medical Sciences, Karaj, Iran .

다 Ali Taherynia, ( ${ }^{\star}$ Corresponding author), Assistant Professor, Emergency Medicine, School of Medicine, Alborz University of Medical Sciences, Karaj, Iran. A.Taherinia@abzums.ac.ir

\section{Abstract}

Background and aims: Hydrogen peroxide is antiseptic due to its oxidizing properties and has anti-bacterial, antiviral and antifungal properties. Chronic exposure to this substance causes coughing, shortness of breath, pulmonary edema and bronchitis. Workers that clean the process line in dairy industries, use hydrogen peroxide as a disinfectant in cleaning the production hall. The purpose of this study is to find the level and type of exposure of workers to hydrogen peroxide and the effects that this pollutant has on the respiratory system of workers.

Methods: The study was conducted as case-control in a dairy industry. 10 workers were selected as the case group who are exposed to hydrogen peroxide and 20 workers as control group. Pollutant sampling in the air was done to determine the amount and distribution rate of hydrogen peroxide. The daily exposure time and work experience was recorded. Respiratory and screening tests were performed by a specialist physician, participants with a pulmonary problem were identified.

Results: The case and control groups had an equal mean age and for the case group, the exposure was higher than the safe limit. Using the statistical analysis, the OR value was 2.52 with a confidence interval of $2.12-10.76$, the results indicate that hydrogen proxide exposure, will increase the risk of pulmonary disease.

Conclusion: Occurrence of respiratory symptoms in exposed workers, has increased in control group. Factors such as work experience and smoking, have also been implicated as factors affecting the risk of disease.

\section{Conflicts of interest: None}

Funding: None

\section{Keywords}

Hydrogen peroxide

Occupational disease

Pulmonary disease

Received: 2019/01/20

Accepted : 2020/03/05 


\section{INTRODUCTION}

Breathing is the most common way that chemicals, especially detergents, enter the body in work places. Most acute and chronic pneumoconiosis can be caused by inhalation of occupational pollutants. Occupational aerosol pollutants, especially gases and chemical vapors, can cause different pulmonary diseases, considering the workers individual differences $(1,2)$.Chronic and acute poisoning by detergents, is a major problem in developing countries and poisoning with some detergent compounds is more common in these countries compared to the more developed countries (3). The toxic effects of being exposed to detergents, have been studied on various organs. However the major route of exposure to these compounds is the inhalation, it has not been studied comprehensively, and the chronic respiratory effects of occupational exposure to these compounds is understudied. Contradictory results for respiratory effects have been reported (4). Hydrogen peroxide is a toxic and irritating liquid that due to oxidizing, has antiseptic, antiviral and antifungal properties (5). Hydrogen peroxide is used to clean dairy and fruit juice packaging equipment (6). The procedure consist of these equipment being immersed in the hydrogen peroxide. Hydrogen peroxide can stimulate the respiratory system. Repeated exposure to this combination may lead to bronchitis, cough, sputum and dyspnea. Long-term exposure to these substances sometimes causes discolored patches and burning sensation on skin. Higher concentrations of hydrogen peroxide can cause red rash and blisters. Inhalation of this substance may cause sore throat, abdominal pain, nausea and vomiting (7). Hydrogen peroxide that is released into the environment, initially vaporizes and gradually decomposes into water and oxygen (8). Cleaning workers at the dairy Industry use hydrogen peroxide to clean the salon. Dairy workers have reported occupational disorders such as asthma, chronic obstructive pulmonary disease, hypertensive pneumonia, chronic bronchitis, and cancer (9). There are no comprehensive study on safe exposure and exposure conditions, individual parameters and other confounding factors that show what proportion of exposure can lead to respiratory disorder in exposed workers. The purpose of this study is finding the amount and type of worker's exposure to hydrogen peroxide, also the long time effect of being exposed to pollutants and the adverse effects it has on the respiratory system of cleaning workers in the dairy industry.

\section{METHODOLOGY:}

This study was planned as a case-control study. Statistical population is workers in a dairy factory. 10 workers were considered as case group exposed to hydrogen peroxide and 20 workers as control group. All the sample subjects had a minimum of 4 years work experience in their work places. Exclusion criteria included people with low work experience, smokers, people with respiratory disease, and age less than 30 and more than 50 years. Hydrogen peroxide was sampled by OSHA VI- 6 standard methods. In this method the sample is collected using a midget fritted-glass bubbler containing $15 \mathrm{~mL} \mathrm{TiO-}$ SO4. An air sampling pump at flow rate of 1.0 $\mathrm{L} / \mathrm{min}$ was used. The pump was properly calibrated so that the volume of the air sample, was determined accurately from the flow rate and time. After sampling for analyte extraction, the samples were transferred to the spectrophotometer vials. Using a spectrophotometer, absorption was measured at $410 \mathrm{~nm}$. From the calibration curve obtained from the standard samples, the amount of contaminants in the sampled analytes was determined. Demographic questionnaire was used to collect information such as age, work experience, underlying disease, hereditary disease and smoking. To obtain the disorder of respiratory system a Vitalograph Compact spirometer was used. All subjects were evaluated by spirometric tests under the assistance of a physician. This test determined the respiratory ability of the subjects. The performance of respiratory system was evaluated by pulmonary volumes and capacities were noted according to the physician opinion. The researches performed in this study, determined the lung Force Vital Capacity (FVC) and the Forced Expiratory Vital capacity at the first second (FEV1). The FEV1 / FVC ratio were measured and determined for all subjects in case and control groups. If this ratio was above $\% .75$, respiratory function was correct. If the ratio is less than $\% 75$ the subject had a respiratory complication. If the ratio was above $\% 75$ but FVC was less than $80 \%$ the subject had a respiratory complication. The results of the questionnaire, spirometry and medical examinations were analyzed by statistical tests. Comparisons between means and nonparametric tests were performed to compare the differences between the means of the case and control groups. Odd $>s$ ratio was calculated and analyzed for both 
Effects of Hydrogen Peroxide on the Respiratory System ...

groups, respiratory health and disease in both groups were noted to make sure of the confidence interval results.

\section{RESULTS}

The case group's mean age was $46.6 \pm 6.9$ and control group $42 \pm 5$. From the comparison of age mean in two groups with definite variance test, it was concluded that the mean age of both groups was equal (95\% confidence). Interfering factors were ineffective according to exclusion criteria, because the underlying and hereditary diseases eliminated in both the case and control groups. By measuring the exposure levels, if the exposure exceeded the 8-hour limit (1ppm), it was identified as an exposed sample, otherwise unexposed. The spirometry test showed that 6 workers out of the case group and 8 workers out of the control group had disorders. The ratio of disordered workers and the workers without any disorders in case and control group was $1.5,0.67$. By statistical analysis and calculating the Odds Ratio, the chances of complication in this workplace due to $\mathrm{H} 2 \mathrm{O} 2$ exposure, was 2.25 times greater in exposed workers. According to confidence interval 2.12-10.76 and OR, it could be said that hydrogen peroxide exposure in the studied population, significantly caused respiratory disorders (95\% confidence). According to the results of the 4-13 years' work experience category, the relationship between exposure and respiratory system disorder was significant and strong. In other words, people with less work experience would suffer fewer side effects. In the category of 13-21 years, this relationship was not detected. There was a significant relationship in the age group of 21 to 30 years category, although, due to the extent of the trust gap, this result cannot be generalized to society. There is also a significant relationship between smoking in the studied groups, but due to the wide range of confidence intervals, this result is not generalizable to the society and it can only be used in case group as a positive factor affecting deliberate pulmonary disorder.

\section{DISCUSSION}

According to the results of this study, the incidence of restricted respiratory symptoms in exposed workers has increased compared to unexposed workers. Result of this study is similar to other studies which were conducted in the field of chronic respiratory symptoms. The respiratory system is a primary target organism for all inhal- able toxins. Accordingly, the function of respiratory system was affected by exposure to hydrogen peroxide by inhalation (10). Long-term exposure to lower levels of pollutants in the workplace may result in chronic disorders such as chronic bronchitis, fibrosis, and lung cancer. Exposure to high concentration of hydrogen peroxide (7 $\mathrm{ppm}$ ), stimulates the upper respiratory system (11). Other symptoms, such as headaches, nausea and diarrhea have been observed among exposed workers (12). No other respiratory symptoms have been studied on workers in this study. Workers are constantly exposed to higher concentrations of hydrogen peroxide in their work place. The concentration of other pollutants, has not been the focus of this study, but it could be concluded that exposure to this substance alone in different ways will eventually lead to respiratory disorders. The results also show that workers exposed to hydrogen peroxide are at risk of respiratory disorder. According to the findings of this study, the cause of lung function defects is unclear when exposed to hydrogen peroxide. Different studies have shown that the exposure, increases production of anionic superoxide in pulmonary arteries (13). However, it can partially be stated that the lung diseases are caused by parenchymal lung disease or neuromuscular disorders (14). The workers in this study did not use appropriate personal protective equipment at their work place. Therefore, hydrogen peroxide uptake may have occurred by any of the inhalation, dermal or even oral ingestion pathways, since, the exact role of either of these pathways in respiratory disorder cannot be presented. Considering the presence of smokers in the study, it is not possible to comment correctly on the extent to which each of these variables has an impact on development of pulmonary morbidity. One of the limitations of this study was simultaneous exposure to several factors, such as hydrogen peroxide and smoking in workers, thus, the respiratory disorder deficits cannot be attributed to exposure to one or a combination of several factors. Another limitation of the study was that the respiratory disease in workers diagnosed by spirometry. According to the studies, the last diagnosis of respiratory disorders will be made by plethysmography which was not possible in this study. It was recommended to determine respiratory disorder in exposed workers, that respiratory function tests be performed, especially when workers' respiratory disorders was increased or when exposed to high concentration contamination. Frequent 
exposure may cause bronchitis, which is associated with coughs and sputum. Long-term exposure can cause temporary whitening of the skin and a burning sensation (11).Long-term exposure with lower levels of contaminants in the workplace may lead to chronic disorders such as chronic bronchitis, lung fibrosis, and lung cancer. Also, long-term side effects due to inhalation of toxic contaminants includes bronchiectasis, bronchiolitis, stable asthma, RADS or asthma caused by irritants, allergies and damage to the nervous system. In the study of Mastrangelo et al. titled exposure by hydrogen peroxide, the results showed that exposure to high concentrations, stimulates the upper respiratory system, which is consistent with the results of this study (8). Result of Rada study on exposure to hydrogen peroxide, showed that being exposed with 7ppm concentrations can cause severe lung irritation. Other symptoms such as headache, nausea, diarrhea, etc. have also been reported (12). The results of this study showed that in concentrations less than $7 \mathrm{ppm}$, these side effects have also occurred. It is recommended that the respiratory function tests be performed before the work begins and after a certain period of time, to determine respiratory complications in workers dealing with repeated exposure, especially when workers' respiratory complaints increases or higher concentrations are reached.

The study did not look at other respiratory symptoms in workers. Exposure of workers in the workshop is continuous and with concentrations higher than the allowed limit. Although the concentration of other contaminants in this plant was not studied in this study, it could be predicted that exposure to this substance alone will lead to pulmonary complications in many different ways. Also, according to the results of the study, it was found that workers exposed to hydrogen peroxide are at higher risk of developing limited lung function. According to the findings of this study, limiting impairments of lung function when exposed to hydrogen peroxide is the main reason that the exposure can affect the respiratory system. In the study of Lisa et al., study of exposure to the inhaled effects of hydrogen peroxide, it was determined that exposure to hydrogen peroxide increases the production of anionic superoxide in the pulmonary vesicles (13). However, to some extent it can be stated that restrictive lung diseases are caused by parenchymal lung diseases or neurological disorders. In Pauluhn study, the effect of being exposed to hydrogen peroxide for 4 weeks, in laboratory rats caused pulmonary complications in the form of $1-2 \mu$ lesions (14). Workers participating in this study did not use appropriate personal protective equipment at work. Therefore, the absorption of hydrogen peroxide may have occurred through any of the inhalation, skin, or even oral swallowing, and it is not possible to provide a theoretical function for the role of either of these pathways. Given the inclusion of smokers in the study, it is not possible to properly comment on the extent to which each of these variables contributes to the development of a restricted pulmonary complication. Lisa's studies have shown that smokers who are also exposed to chemicals have more respiratory symptoms and spirometry disorders than non-smokers (13). One of the limitations of this study was that because there was simultaneous exposure to several factors such as high levels of hydrogen peroxide and smoking in workers, lung defects could not be attributed to exposure to one or a combination of several factors. Another limitation of the study was that the restrictive lung disease in workers was diagnosed by spirometry. According to the sources, the final diagnosis of restricted lung disease will be made by plethysmography, which was not possible in this study.

\section{ACKNOWLEDGMENT}

We would like to thank the research assistant of Alborz University of Medical Sciences, Student research committee and supervisor of this project at Alborz University of Medical Sciences, as well as the managers, workers and colleagues.

How to cite this article:

Jamshid Rahimi, Ali Taherynia. Effects of Hydrogen Peroxide on the Respiratory System of Dairy Industry Workers. Iran Occupational Health. 2021 (01 Dec);18:23.

*This work is published under CC BY-NC 4.0 licence 

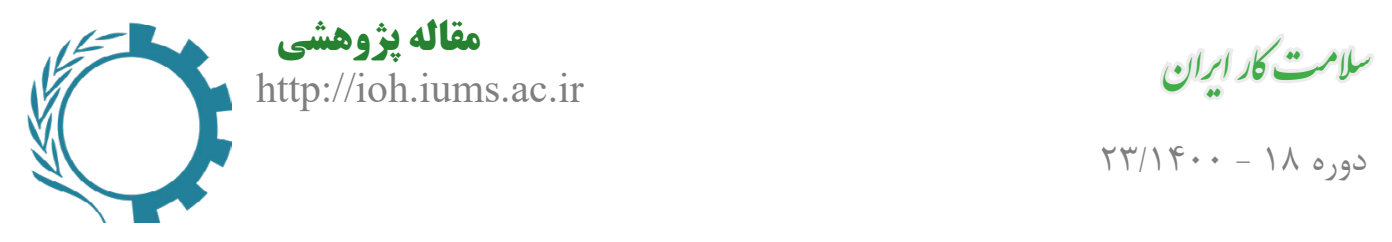

rr/IF... - IN

\section{بررسى اثر آب اكسيزنه بر سلامت دستكاه تنفسى كارتران كار خانه لبنيات}

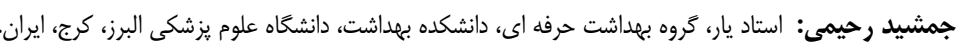

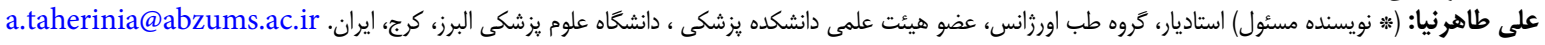

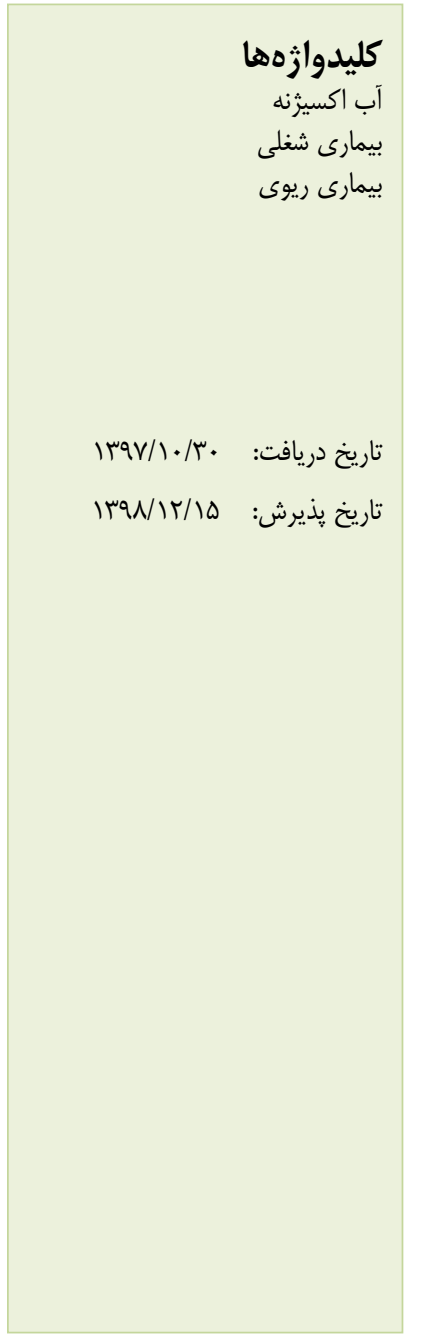

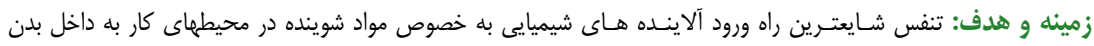

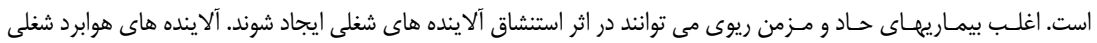

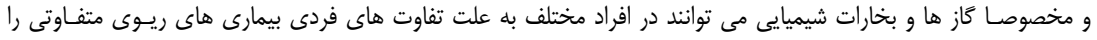

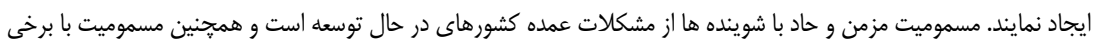

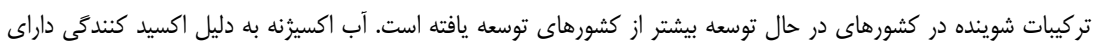

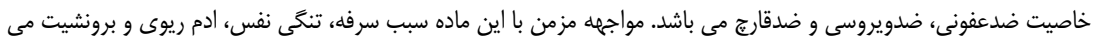

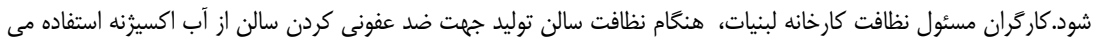

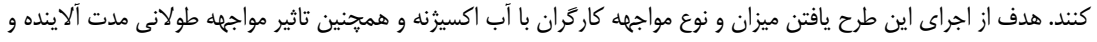

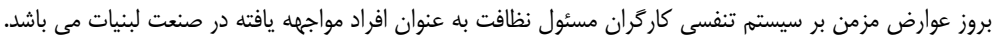

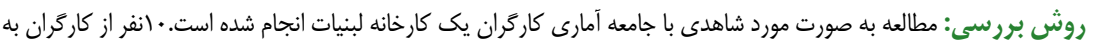

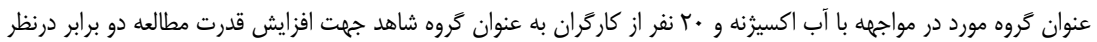

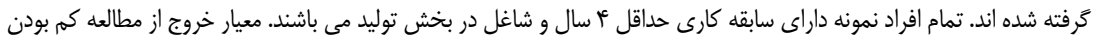

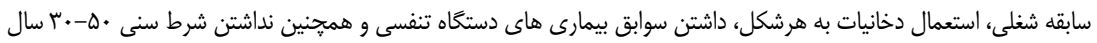

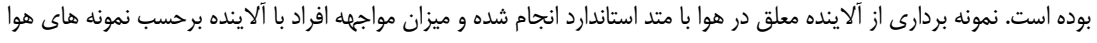

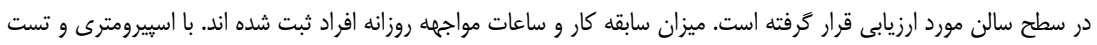

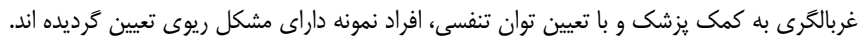

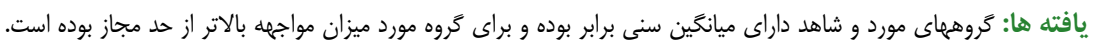

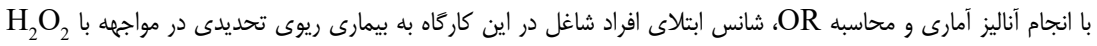

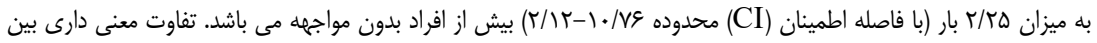

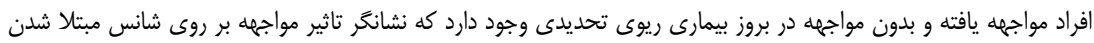
بله بيمارى ريوى مى باشد.

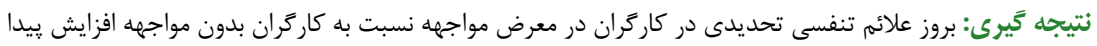

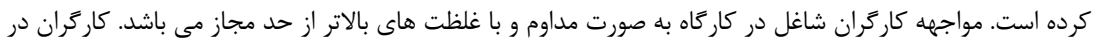

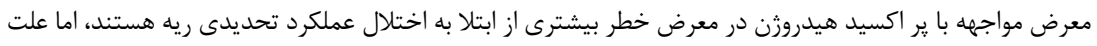

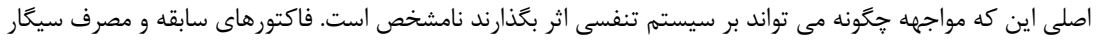
نيز به عنوان عوامل موثر در ايجاد بيمارى نقش دانه داشته اند. تنعارض منافع: كزارش نشده /ست.

شيوه استناد به اين مقاله: Jamshid Rahimi, Ali Taherynia. Effects of Hydrogen Peroxide on the Respiratory System of Dairy Industry Workers. Iran Occupational Health. 2021 (01 Dec);18:23. 
و به دليل ايجاد واكنش شيميايى و توليد كاز مى تواند

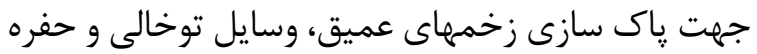

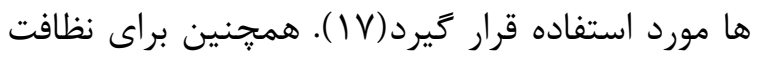

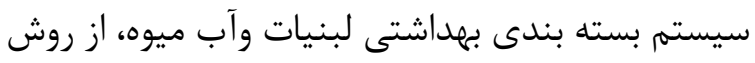

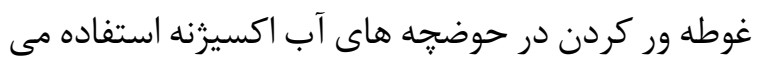

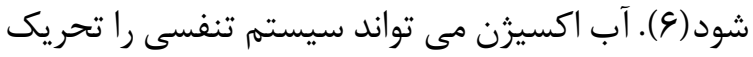

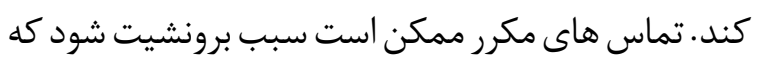

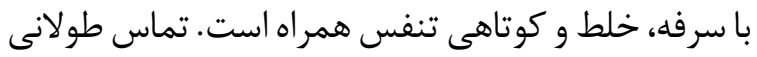

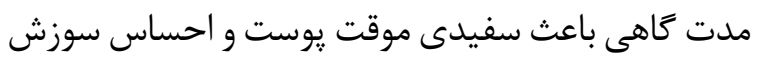

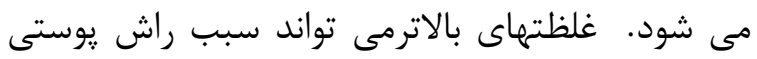

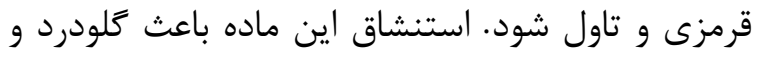

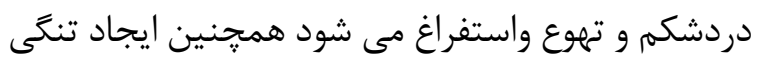

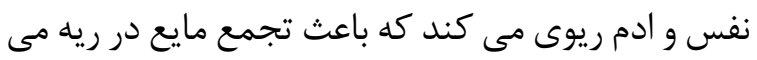

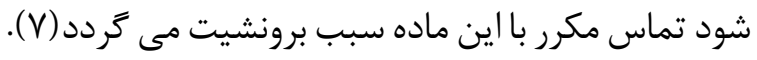

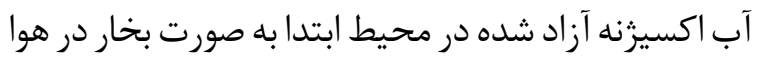

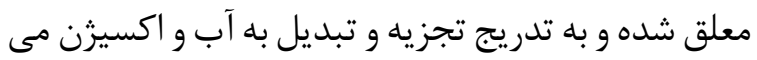

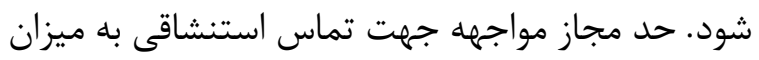

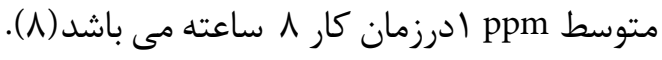

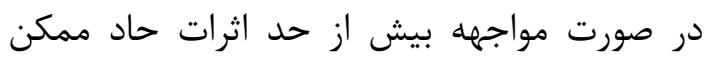

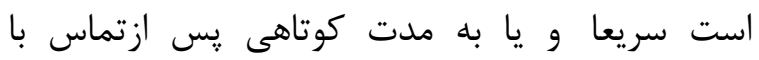

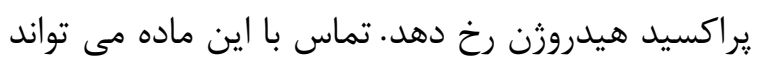

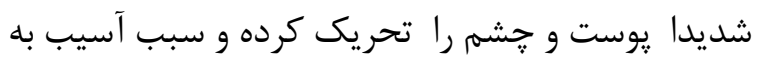

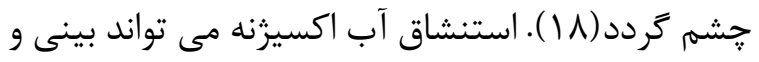

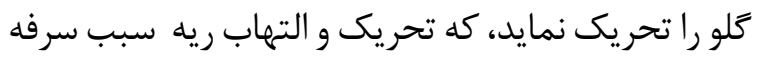

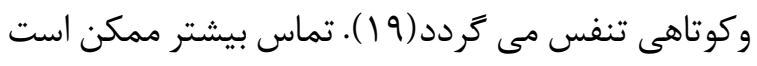

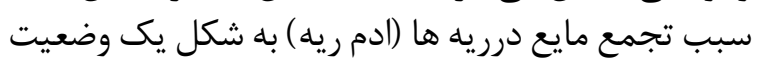

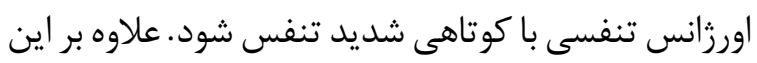

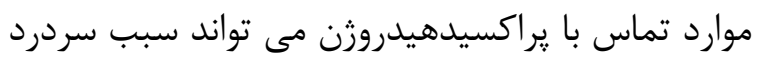

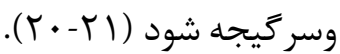

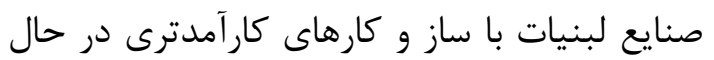

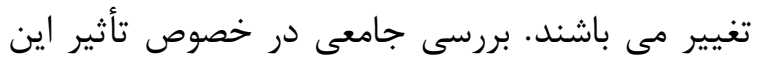

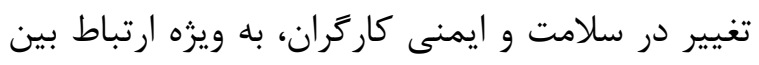

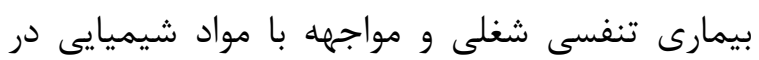

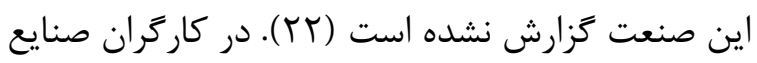

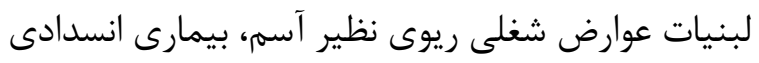

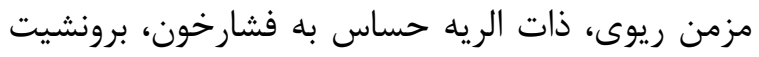

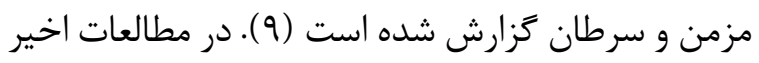

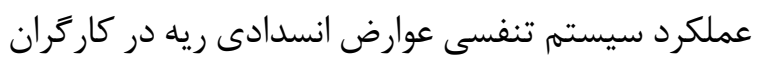

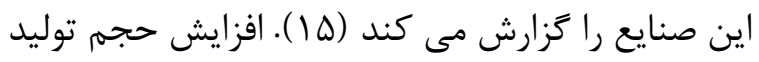

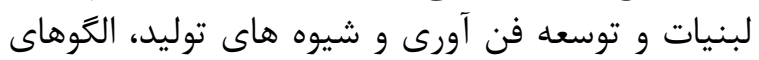

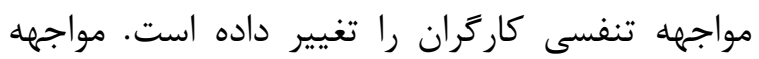

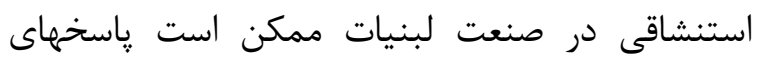

مقالمها

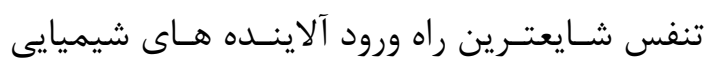

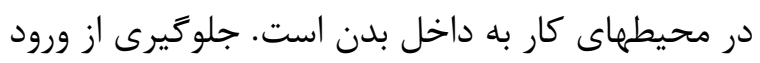

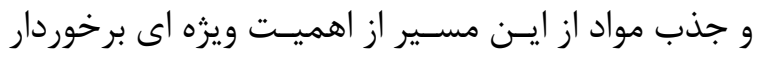

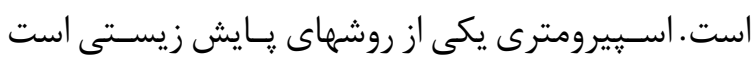

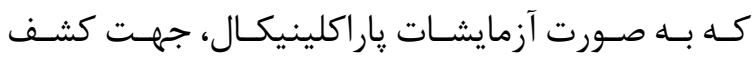

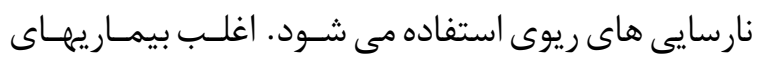

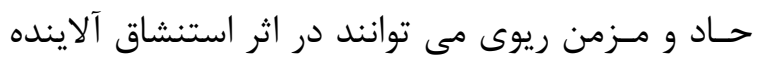

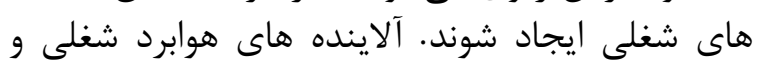

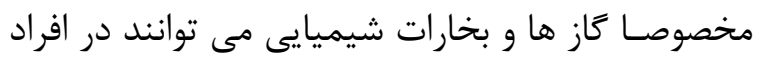

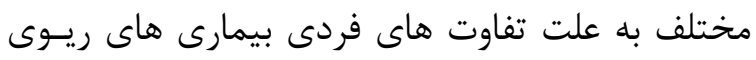

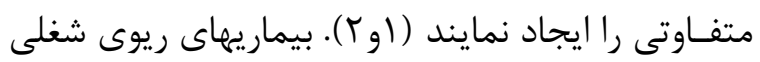

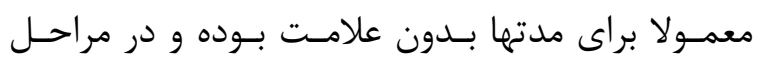

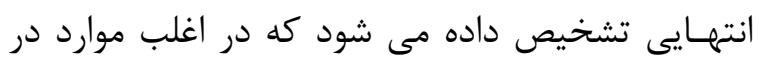

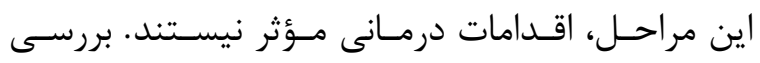

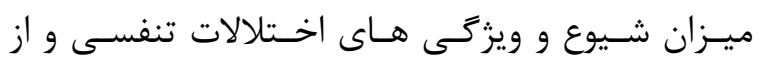

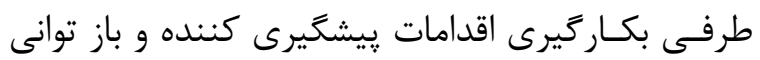

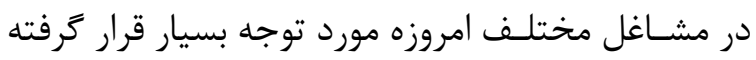

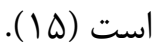

مسموميت مزمن و حاد با شوينده ها از مشكلات عمده

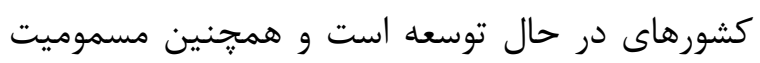

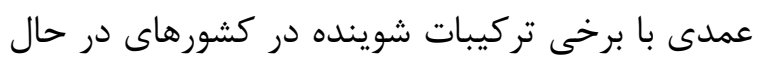

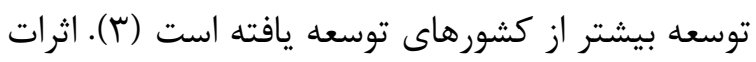

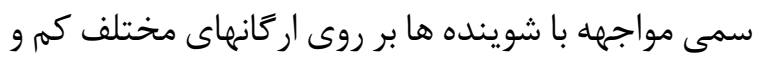

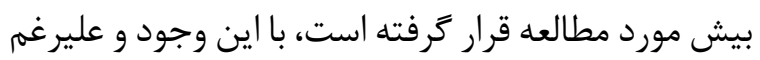

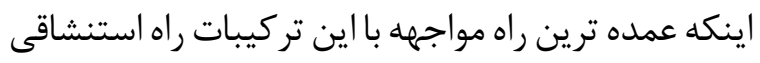

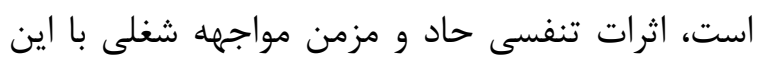

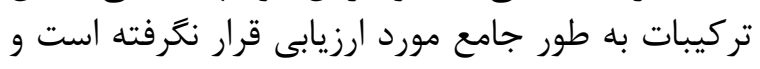

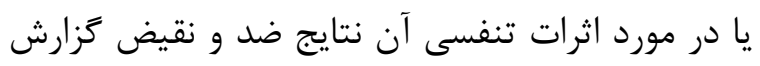

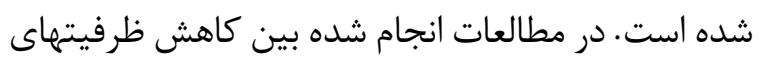

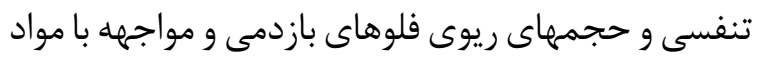

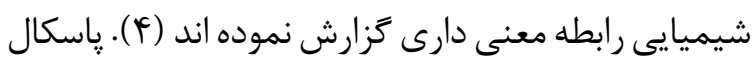

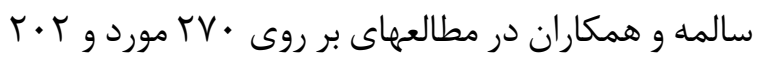

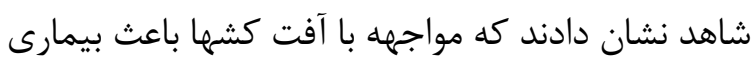
آسم مى شود (19 آنان ). آب اكسيزنه خالص

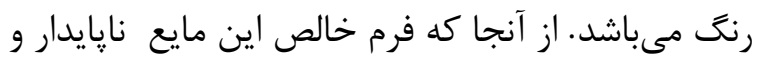

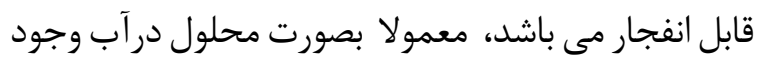

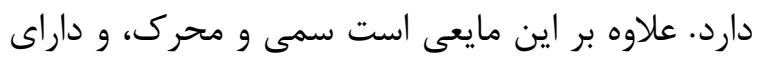

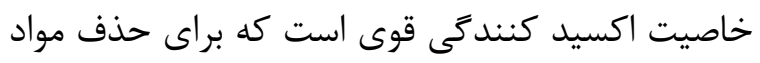

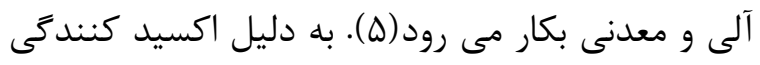

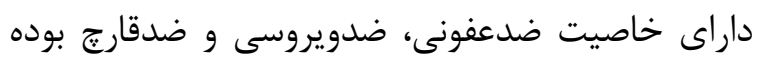


يراكندگى توسط سنجش هاى آزمايشگاهى مطابق با متد

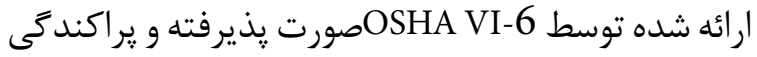

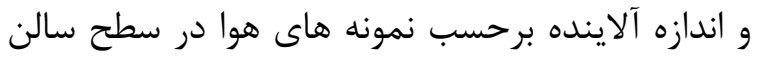

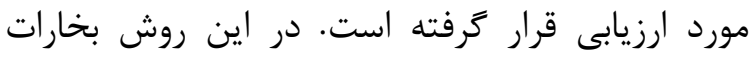

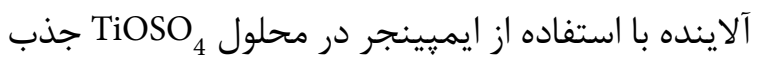

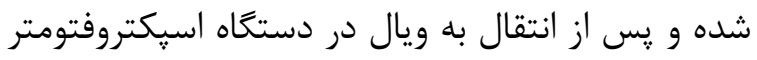

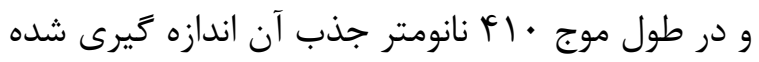

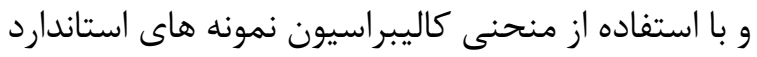

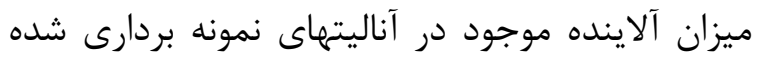

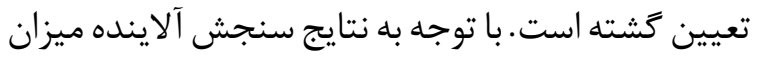

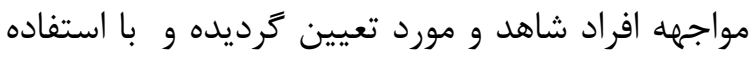

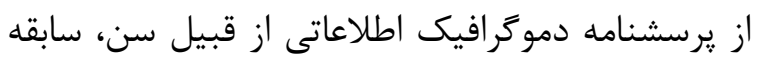

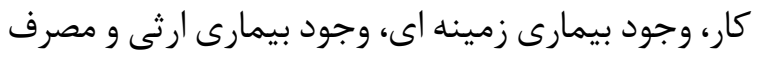
دخانيات جمع آورى شده نداست زينه اس،

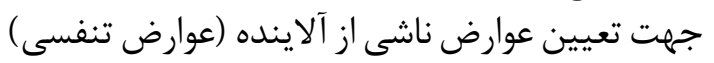

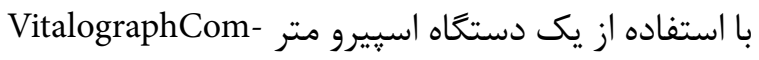

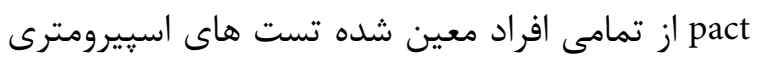

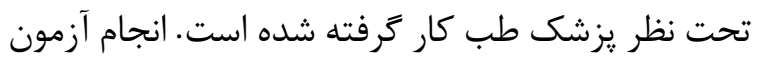

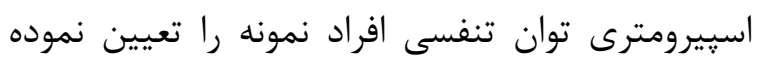

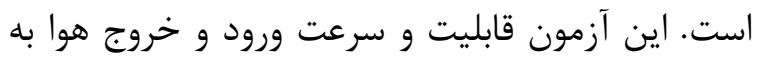

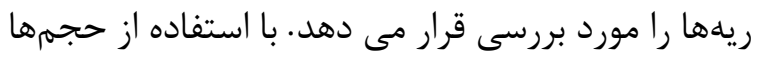

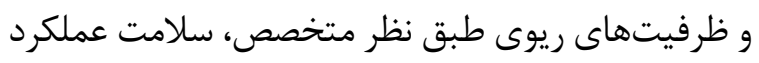

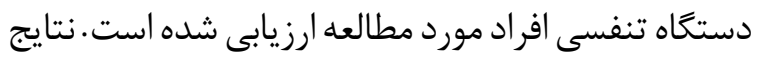

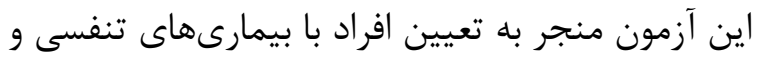

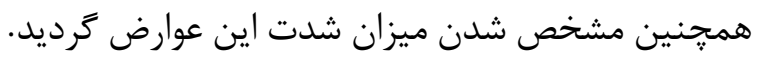

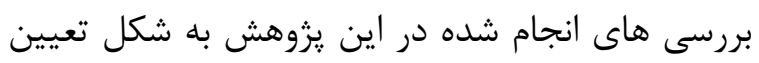

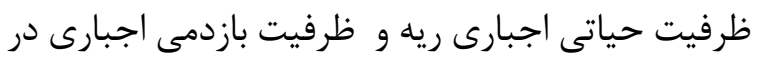

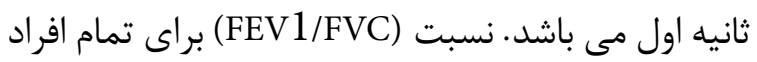

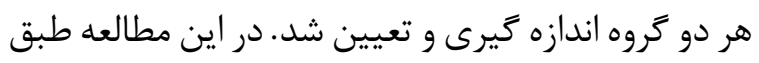

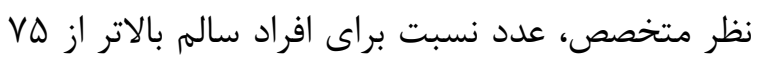

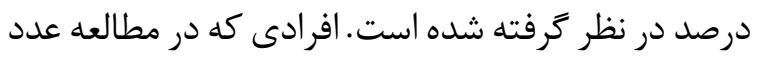

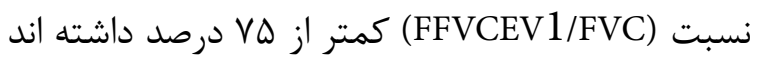

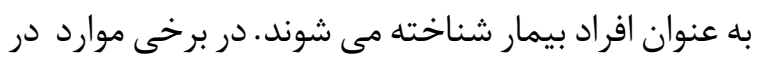

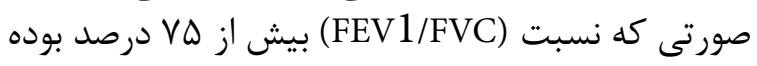

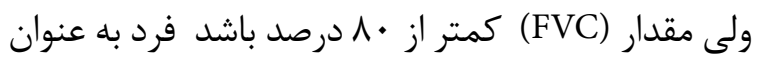
بيمار درنظر كرفته مى شود.

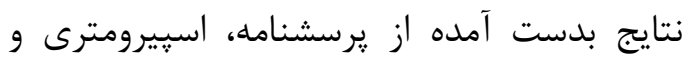

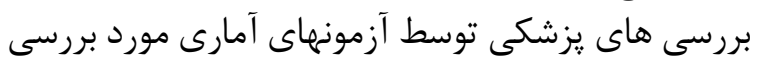

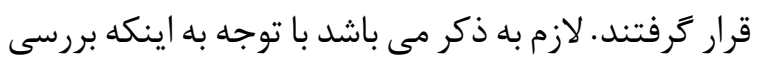

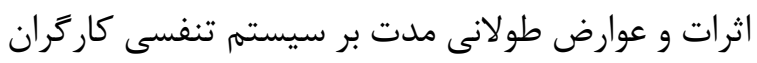

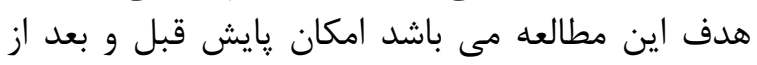

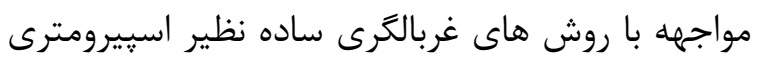

التهابى متفاوتى در مواجها حاد و مزمن ايجاد كند.

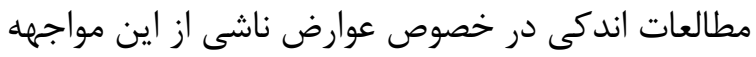

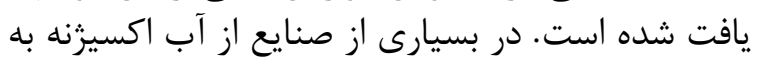

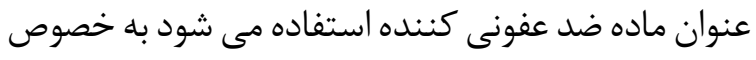

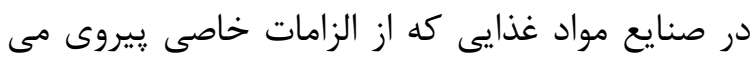

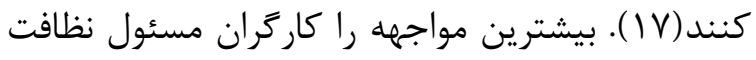

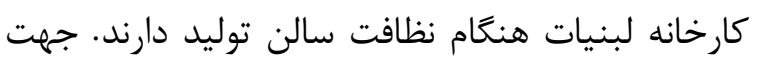

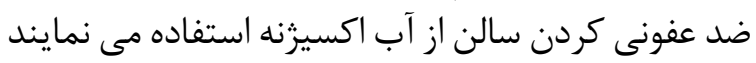

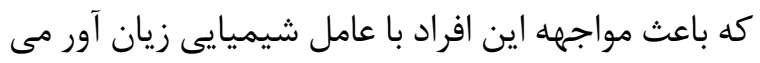

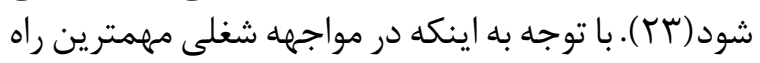

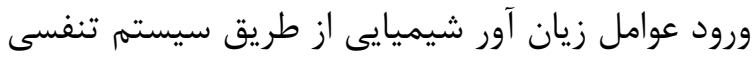

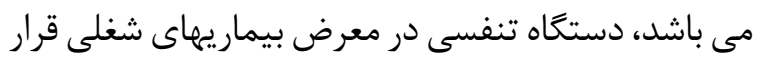

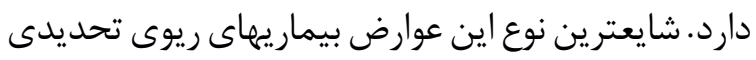

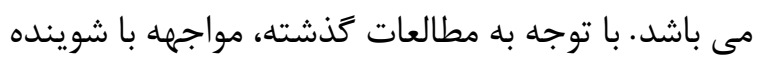

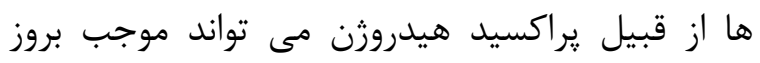

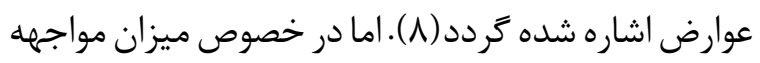

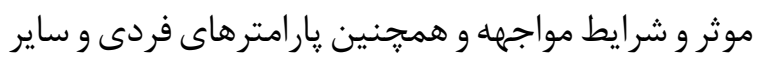

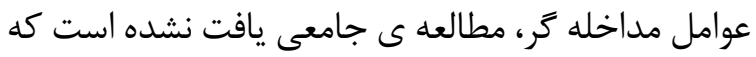

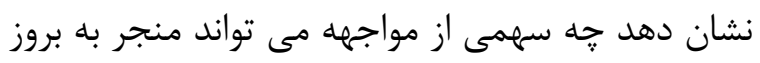

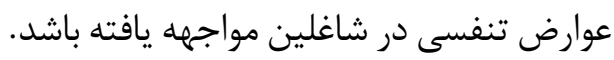

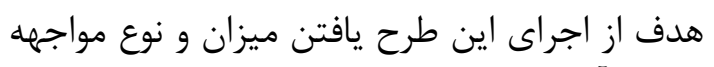

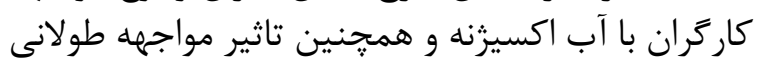

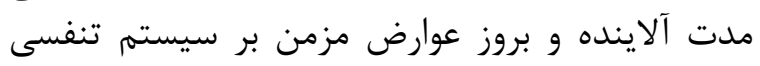

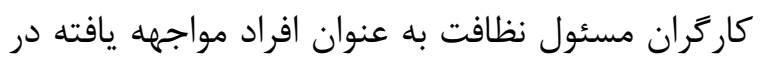
صنعت لبنيات مى باشد.

$$
\text { روش كار }
$$

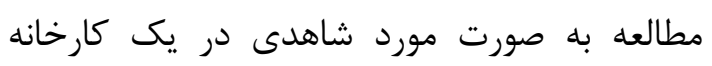

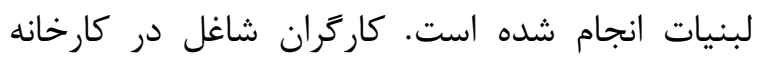

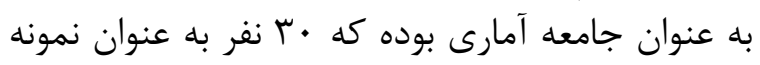

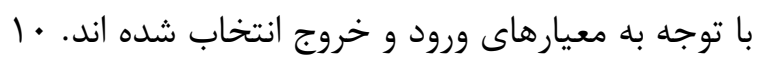

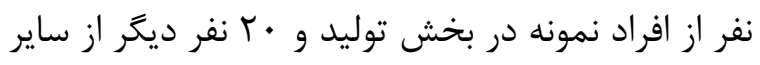

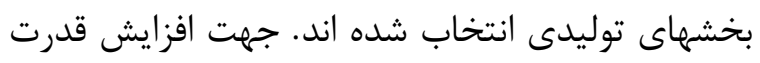

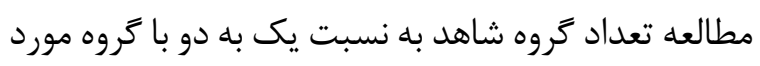

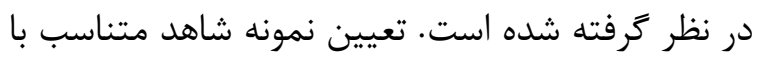

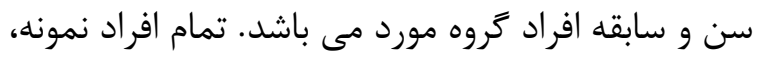

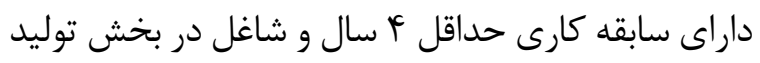

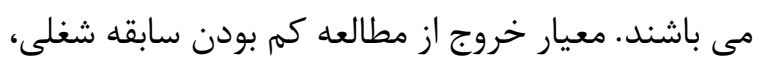

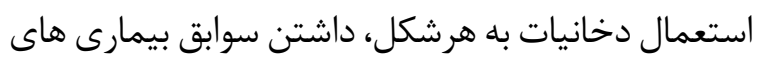

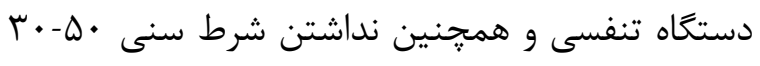

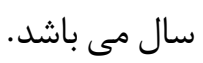
نمونه بردارى از آلاينده جهت تعيين ميزان و نحوهى 
هاى زمينه اى و ارثى در دو گروه مورد و شاهد به عنوان فاكتورهاى بدون تاثير در نظر ترفته شده است. ميزان مواجها با بان

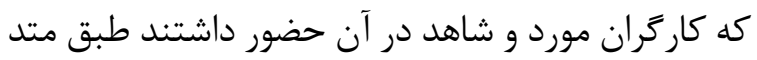

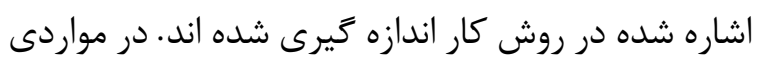

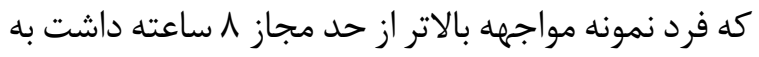

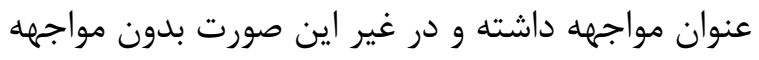

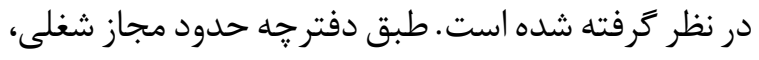

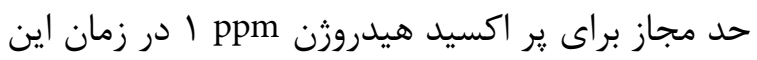

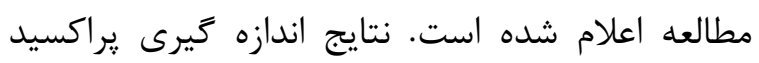

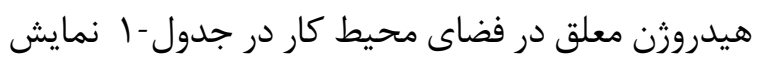

$$
\text { داده شده است. }
$$

با استفاده از تست اسييرومترى غربالكرى ميزان

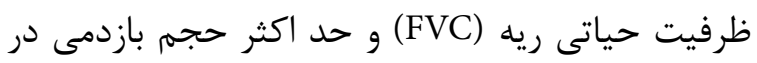

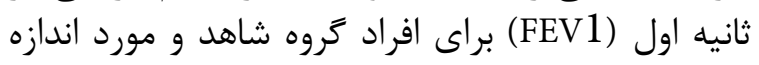

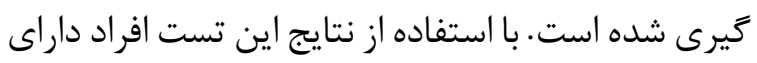

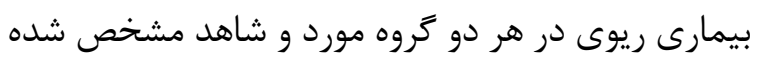

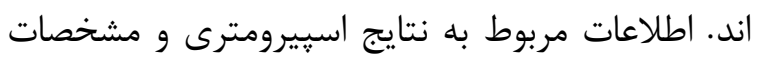

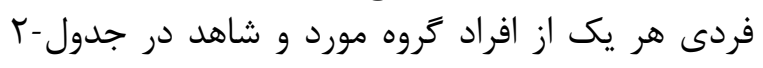

وجود نداشته است. در اين مطالعه سعى بر اين بود كه

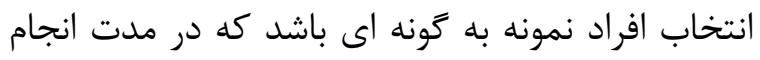

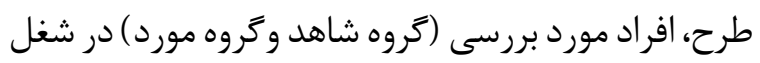
و محيط كار جابجايى نداشته باشند.

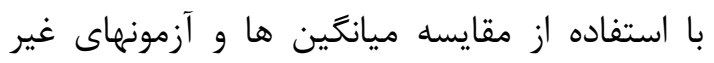

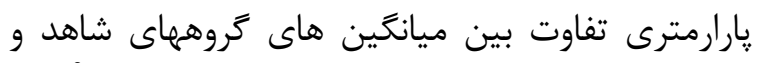

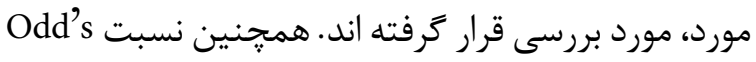

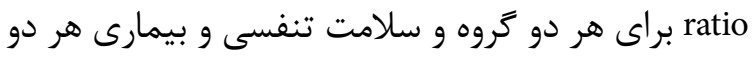

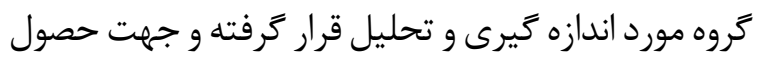
اطمينان از نتايج فاصله اطمينان نيز محاسبه كرديد ترديد است

نتايج

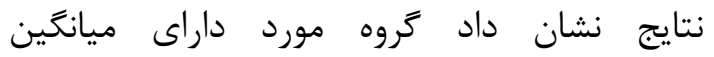

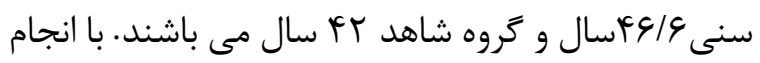

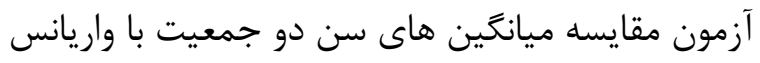

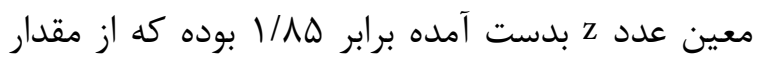

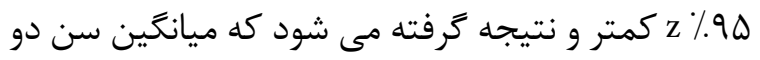

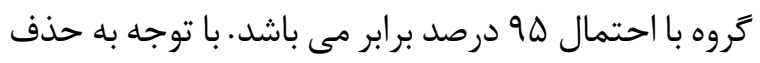

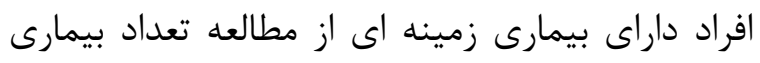

جدول (. نتايج اندازه كيرى

\begin{tabular}{|c|c|c|c|}
\hline مقايسه با استاندارد & مقدار (ppm) & ساعات مواجهله & محل \\
\hline كمتر از حد مجاز & $\cdot / r$ & $v$ & توليد ابتداى خط \\
\hline بالاتر از حد مجاز & $1 / 9$ & v & توليد بخش تركيب \\
\hline كمتر از حد مجاز & $\cdot / \Lambda$ & v & توليد هم زن بسته \\
\hline بالاتر از حد مجاز & $1 / \pi$ & $\wedge$ & توليد هم زن باز \\
\hline بالاتر از حد مجاز & $1 / 9$ & $\wedge$ & قالب زنى \\
\hline كمتر از حد مجاز & $\cdot / V$ & 9 & بسته بندى \\
\hline بالاتر از حد مجاز & $1 / 1$ & 9 & شستشوى قالب \\
\hline بالاتر از حد مجاز &.$/ 9$ & 9 & خشك كن قالب \\
\hline بالاتر از حد مجاز & $1 / 1$ & $\wedge$ & شستشوى ظروف \\
\hline بالاتر از حد مجاز &.$/ 4$ & $\wedge$ & انبار محصول \\
\hline
\end{tabular}

جدول Y الف. مشخصات و نتايج اسييرو مترى گروه مورد

\begin{tabular}{|c|c|c|c|c|c|c|c|}
\hline بيمارى & مواجهه & FEV1/FVC\% & FEV1 & FVC & سابقه & سن & رديف \\
\hline بيمار & $1 / 9$ & $1 . \mu$ & $v^{e}$ & ve & 9 & ra & 1 \\
\hline سالم & $1 / 8$ & $1 \cdot 1$ & ve & Nr & 19 & $\Delta$. & r \\
\hline 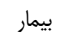 & $1 / r$ & $1 \cdot 0$ & VQ & va & $M$ & is & r \\
\hline 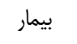 & $1 / r$ & $1 \cdot v$ & $v^{e}$ & va & TV & $\hat{~ i \Lambda}$ & i \\
\hline 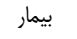 & $1 / 8$ & 99 & $v^{c}$ & $v^{m}$ & 15 & is & $\Delta$ \\
\hline بيمار & $1 / \Lambda$ & q. & va & $n$ & זr & $\Delta F$ & 9 \\
\hline سالم سم & $1 / 1$ & $1 .$. & $\wedge$. & $\wedge \cdot$ & M & 19 & v \\
\hline 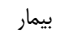 & $1 / 1$ & $1 \cdot 0$ & va & $\wedge \mu$ & ir & is & $\wedge$ \\
\hline سالم & $1 / 1$ & $1 \cdot v$ & $v^{\mu}$ & $\mathrm{v} \wedge$ & rq & $\Delta V$ & 9 \\
\hline سالم & $1 / 1$ & $1 \ldots$ & $\wedge 1$ & $\wedge 1$ & 10 & q & 1. \\
\hline- & $1 / 4$ & $1 . r / 4$ & $V \varepsilon / \Delta$ & $V N / T$ & $1 N / 1$ & $\mid \varphi / 8$ & ميانكين \\
\hline- & $\cdot / T \Lambda$ & $\Delta / \uparrow^{c}$ & $r / 9$ & $r / \Lambda$ & $8 / 9$ & $8 / 9$ & انحرف معيار \\
\hline
\end{tabular}


بررسى اثر آب اكسيرنه بر سلامت دستخاه تنفسى كاركران كارخانه لبنيات

جدول ب ب. مشخصات و نتايج اسيبرو مترى گروه شاهد

\begin{tabular}{|c|c|c|c|c|c|c|c|}
\hline بيمارى & مواجهه & FEV1/FVC\% & FEV1 & FVC & سابقه & سن & رديف \\
\hline سالم & . & q. & ᄉ. & 19 & 11 & rq & 1 \\
\hline سالم & $\cdot / \Lambda$ & १६ & 11 & $\Delta F$ & 10 & r. & $r$ \\
\hline بيمار & $\cdot / \Lambda$ & $11 \mathrm{~V}$ & 11 & 99 & 11 & kr & $r$ \\
\hline سالم &.$/ V$ & १९ & 11 & $\Delta F$ & F & $r$. & F \\
\hline سالم & $\cdot / V$ & $9 f$ & 11 & $N G$ & 10 & f. & $\Delta$ \\
\hline بيمار & $\cdot / V$ & זrו & $\wedge$ & 98 & IV & eq & 9 \\
\hline سالم &.$/ \mu$ & १ & 11 & $\wedge \mathrm{V}$ & 11 & r & v \\
\hline سالم & . & $9 \Delta$ & 11 & $\Lambda \Delta$ & $r r$ & Q) & $\wedge$ \\
\hline سالم &.$/ 4$ & $9_{\Delta}$ & 11 & $\Lambda \Delta$ & $r$ & $\mu r$ & 9 \\
\hline ساله &.$/ 4$ & 1.1 & $\wedge r$ & 1) & $\pi$ & i) & 1. \\
\hline بيمار &.$/ 4$ & $I T \Lambda$ & $\wedge \mu$ & 90 & 18 & f. & 11 \\
\hline سالم & $\cdot / \mu$ & 91 & ^ & $\Lambda \Delta$ & IV & et & ir \\
\hline سالم & $\cdot / \Lambda$ & $1 .$. & $\wedge$ & $\Delta r$ & 11 & $\mu$ & rו \\
\hline سالم & $\cdot / 1$ & १V & $\Lambda \mu$ & $N \varepsilon$ & 9 & f. & if \\
\hline بيمار & $\cdot / 4$ & 114 & ^ & $v^{m}$ & 19 & is & 10 \\
\hline بيمار & $\cdot / V$ & 1.9 & $\wedge i$ & w & 10 & rq & 19 \\
\hline سالم & $\cdot / V$ & १V & $\wedge f$ & $\wedge \vee$ & 19 & Qr & IV \\
\hline بيمار & $\cdot / r$ & $q$. & $\wedge f$ & $\mathrm{v} \wedge$ & v & f. & M \\
\hline ساله & 1 & १९ & $\wedge \vee$ & $9 f$ & 19 & et & 19 \\
\hline سالم & 1 & liv & १) & 111 & $r$. & $\Delta$. & r. \\
\hline- &.$/ 8$ & $1 . r / \%$ & $\Lambda \tau / V$ & $\Lambda r / v$ & $10 / T$ & et & ميانگين \\
\hline- & $\cdot / T \Delta$ & $11 / 4$ & $\Gamma / \Delta \Delta$ & $1 . / r$ & $\Delta / \Lambda$ & $\Delta$ & انحرف معيار \\
\hline
\end{tabular}

جدول ". تعداد مواجهه دو كروه

\begin{tabular}{|c|c|c|}
\hline كروه شاهد & كروه مورد & \\
\hline$\wedge$ & 9 & بيمار \\
\hline$\pi$ & i & سالم \\
\hline
\end{tabular}

با توجه به زستره بدست آمده و همجنين

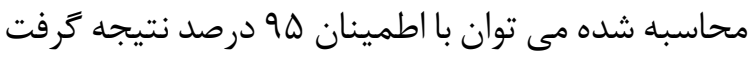
كه مواجهه با در $\mathrm{H}_{2} \mathrm{O}_{2}$ تحديدى ارتباط معنى دارى دارد.

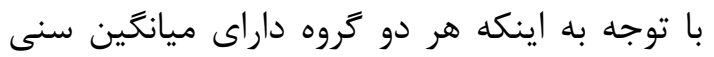

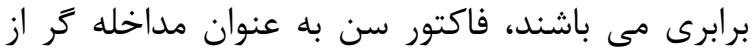

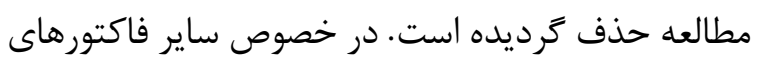

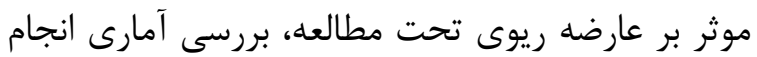

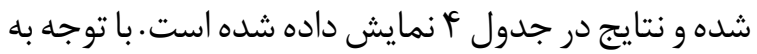

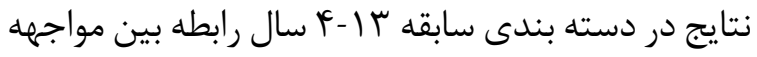

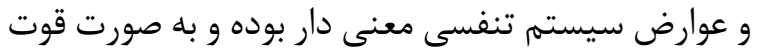

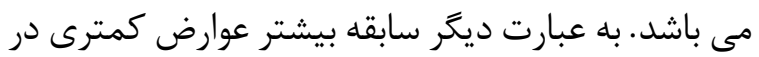

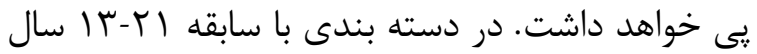

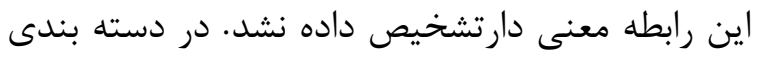

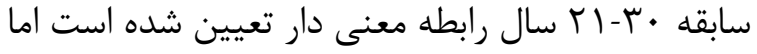

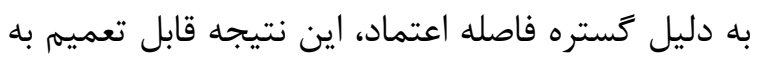

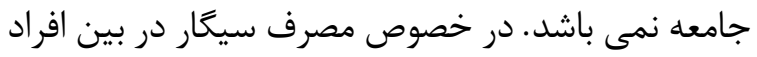

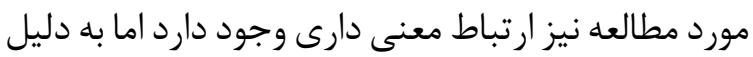

الف (گروه مورد) و ب (كره شاهد) نمايش داده شده است.

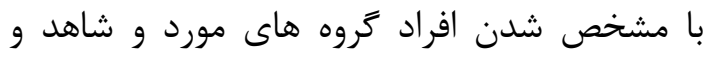

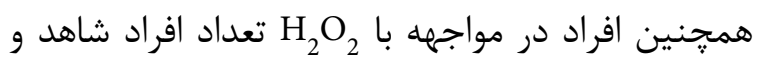

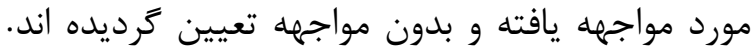

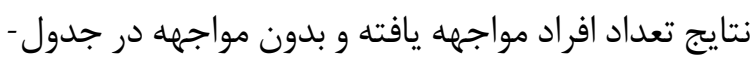
س نمايش داده شده است.

Odd's Ratio مجهت بررسى تاثير مواجهه مقدار محاسبه كرديده كه به شرح زير مى باشد:

$\mathrm{OR}=\frac{6 \times 12}{8 \times 4}=2.25$

با توجه به مقدار OR شانس ابتلاى افراد شاغل در اين كار كاه به بيمارى ريوى تحديدى درى در مواجها

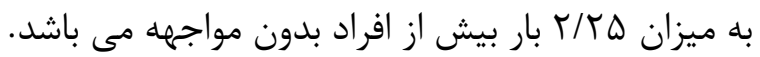

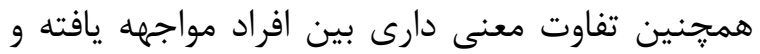

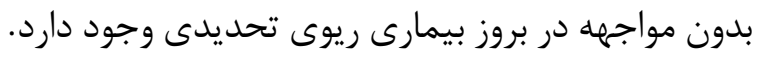

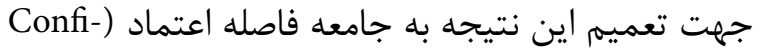

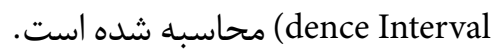

$C I=2.12-10.76$

Iran Occupational Health. 2021 (01 Jan);18: * 
جدول ع. تعداد بيماران در كروههاى مورد و شاهد به تفكيك سن و سيكار و ...

\begin{tabular}{|c|c|c|c|c|}
\hline CI95\% & OR & شاهد & مورد & متغير \\
\hline$T / 1+-1 T / V 7$ & T/TO & $\wedge$ & 7 & سوابقه \\
\hline$+/ 1-r / 9$ & $+/ T$ & $v$ & 1 & $1 w-\varepsilon$ \\
\hline •|乏-r|/0 & 1 & 1. & 0 & rו-וT \\
\hline • & $r / \varepsilon$ & $r$ & r & $r+-r^{\prime}$ \\
\hline */Tr-T & $r / V$ & $r$ & $\varepsilon$ & سيخًار \\
\hline- & - & + & + & بيمارى ارثى \\
\hline- & - & 1 & • & بيمارى زمينه اى \\
\hline + $/ 0-r o / q$ & $1 / r$ & $\checkmark$ & $\varepsilon$ & استفاده از ماسك \\
\hline
\end{tabular}

با نتاج اين مطالعه مى باشد(N). در مطالعه رادا نشان

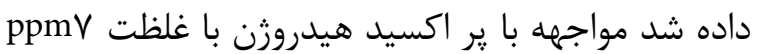

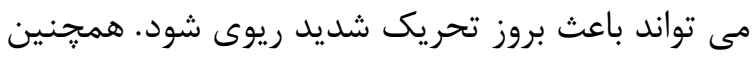

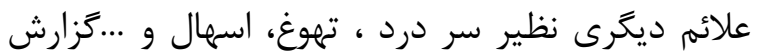

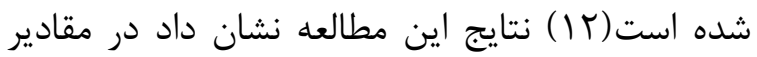

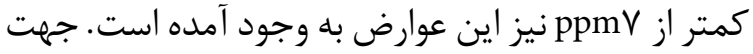

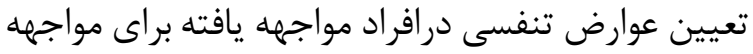

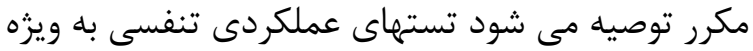

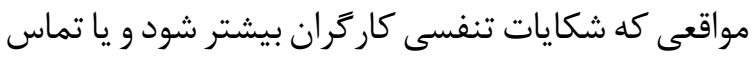

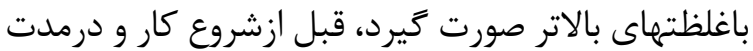
زمان معينى يس ازآن آنجام يذيردي.

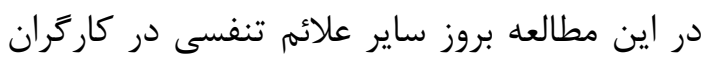

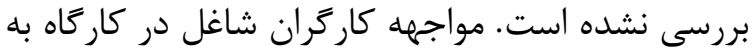

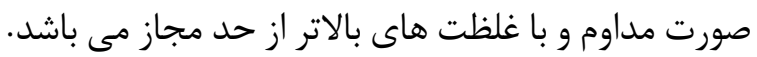

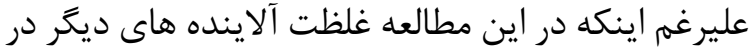

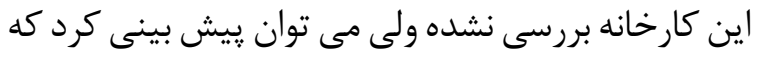

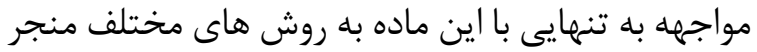

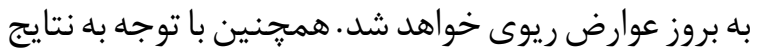

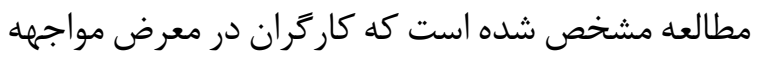

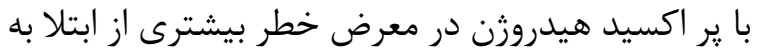

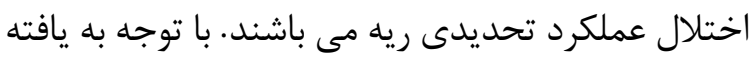

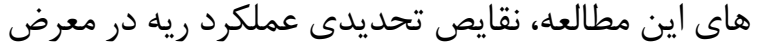

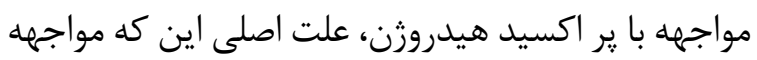

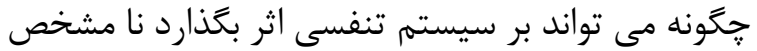

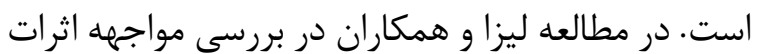

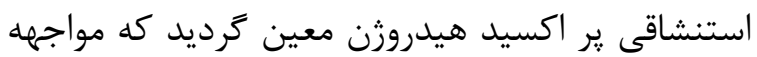

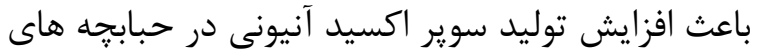

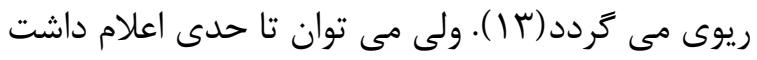

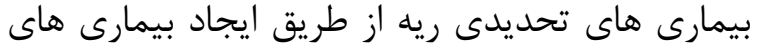

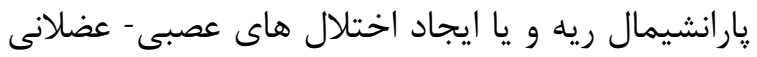

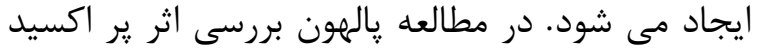

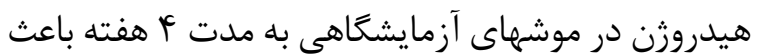

گَستره وسيع فاصله اعتماد، اين نتيجه قابل تعميم به

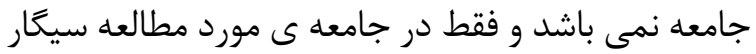

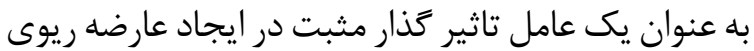

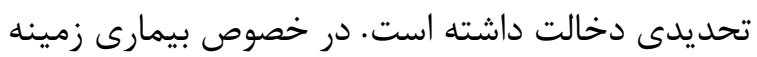

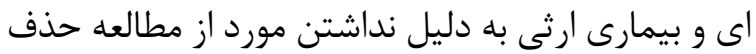

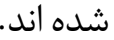

بصث با توجه به نتايج به دست آمده در اين مطالعه بروز

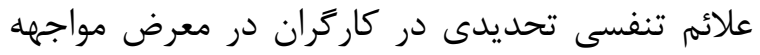

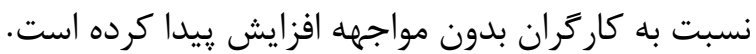

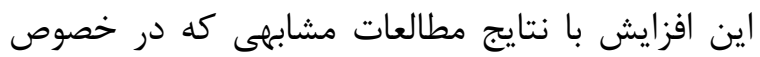

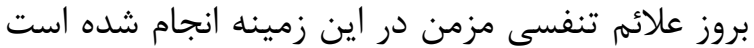

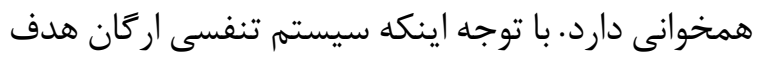

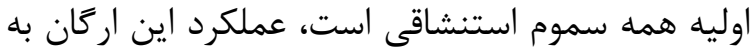

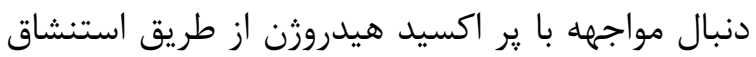

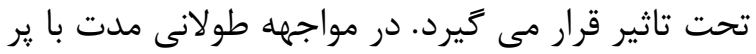

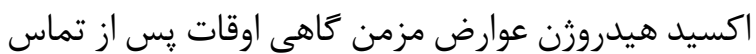

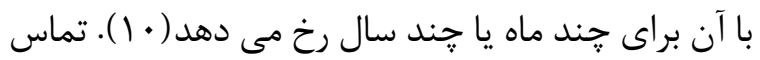

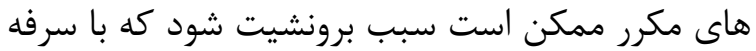

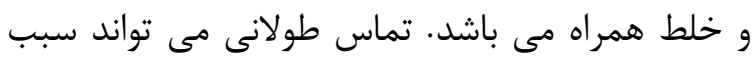

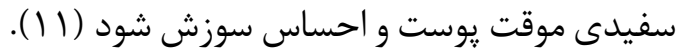

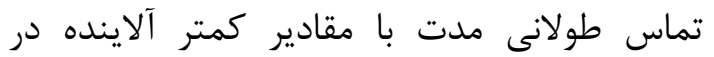

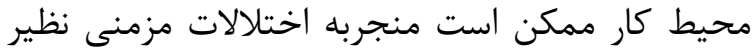

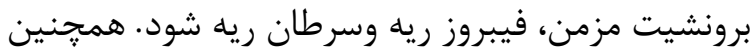

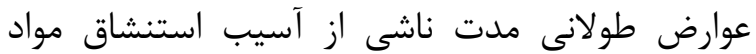

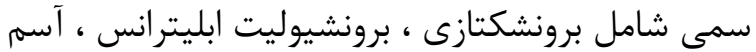

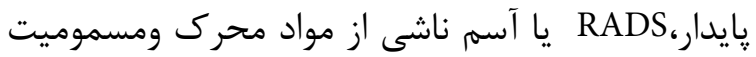

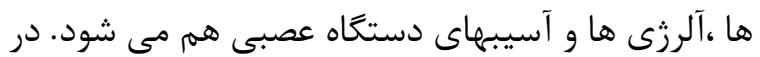

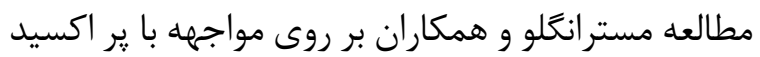

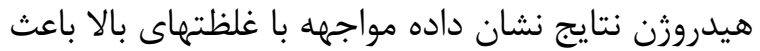

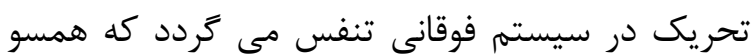


بررسى اثر آب اكسيرنه بر سلامت دستخاه تنفسى كاركران كارخانه لبنيات

paraquat, among workers in the Western Cape. Occupational and Environmental Medicine. 1999 Jun 1;56(6):391-6.

5. Stone JR, Yang S. Hydrogen peroxide: a signaling messenger. Antioxidants \& redox signaling. 2006 Mar 1;8(3-4):243-70.

6. Boveris A, Chance B. The mitochondrial generation of hydrogen peroxide. General properties and effect of hyperbaric oxygen. Biochemical Journal. 1973 Jul 15;134(3):707-16.

7. French LK, Horowitz BZ, Mckeown NJ. Hydrogen peroxide ingestion associated with portal venousgas and treatment with hyperbaric oxygen: A case seriesand review of the literature. Clinical toxicology. 2010 Jul 1;48(6):533-8.

8. Mastrangelo G, Zanibellato R, Fadda E, Lange JH, Scoizzato L, Rylander R. Exposure to hydrogen peroxide and eye and nose symptoms among workers in a beverage processing plant. Annals of occupational hygiene. 2009 Mar 1;53(2):161-5.

9. Reynolds SJ, Nonnenmann MW, Basinas I, Davidson M, Elfman L, Gordon J, Kirychuck S, Reed S, Schaeffer JW, Schenker MB, Schlünssen V. Systematic review of respiratory health among dairy workers. Journal of agromedicine. 2013 Jul 3;18(3):219-43.

10. Weitzman SA, Weitberg AB, Stossel TP, Schwartz J, Shklar G. Effects of hydrogen peroxide on oral carcinogenesis in hamsters. Journal of Periodontology. 1986 Nov;57(11):685-8.

11. Watt BE, Proudfoot AT, Vale JA. Hydrogen peroxide poisoning. Toxicological reviews. 2004 Mar;23(1):51-7.

12. Rada B, Leto TL. Characterization of hydrogen peroxide production by Duox in bronchial epithelial cells exposed to Pseudomonas aeruginosa. FEBS letters. 2010 Mar 5;584(5):917-22.

13. Golshan M, MEER AA, Mohammadzadeh Z, Kyani Y, Loghmanian L. Prevalence of asthma and related symptoms in school-aged children in zarinshahr, Iran.

14. Pauluhn J. Retrospective analysis of 4-week inhalation studies in rats with focus on fate and pulmonary toxicity of two nanosized aluminum oxyhydroxides (boehmite) and pigment-grade iron oxide (magnetite): the key metric of dose is particle mass and not particle surface area. Toxicology. 2009 May 17;259(3):140-8.

15. Vil'ians' ka OM, Rodionova VV. Characteristics of broncho-pulmonary diseases in workers employed in unsafe working environment. Likars' ka sprava. 2006 Jan 1(1-2):34-8.

16. Harbison RD, Bourgeois MM, Johnson GT. Hamilton and hardy's industrial toxicology. John Wiley \& Sons; 2015 Apr 13.

17. Wang J, Lin Y, Chen L. Organic-phase biosensors for monitoring phenol and hydrogen peroxide in pharmaceutical antibacterial products. Analyst. 1993 Jan 1;118(3):277-80.

18. Zmijewski JW, Lorne E, Zhao X, Tsuruta Y, Sha Y, Liu G, Abraham E. Antiinflammatory effects of hydrogen peroxide in neutrophil activation and acute lung injury. American journal of respiratory and critical care medicine. 2009 Apr 15;179(8):694-704.

19. Soltani HA, Zeraatkari K, Veisyraygani A, Soleimani B. Cidex, savlon and hydrogen peroxide: which of them is more effective in disinfection of ventilator tubes: A-110. European Journal of Anaesthesiology (EJA). 2004 Jun 1;21:27-8.

20. Toth KM, Clifford DP, Berger EM, White CW, Repine JE. Intact human
بروز عوارض ريوى به صورت ضايعات ك-1 إ ميكرومترى

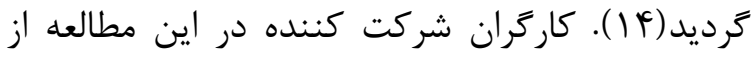

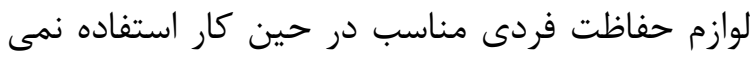

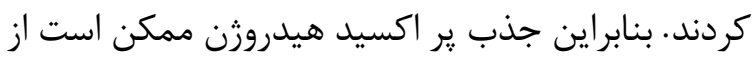

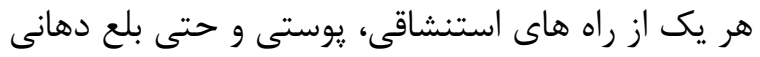

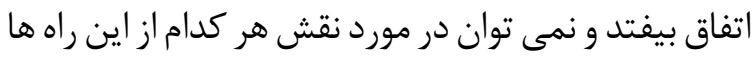

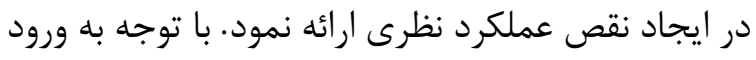

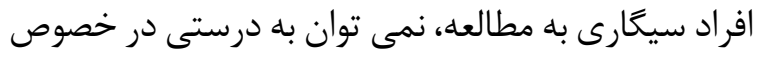

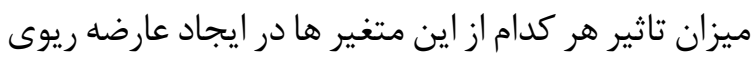

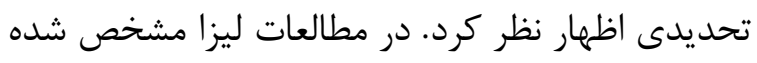

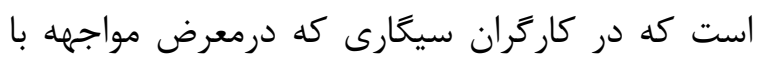

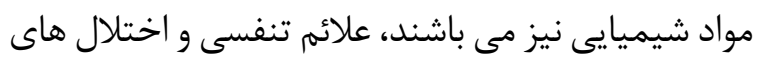

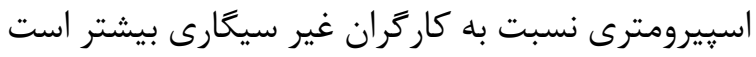

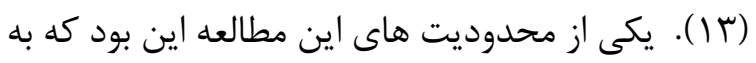

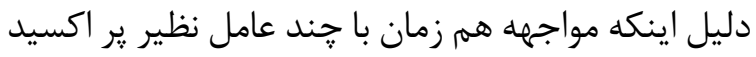

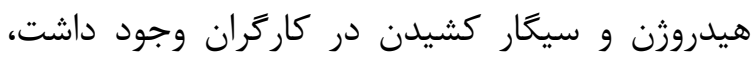

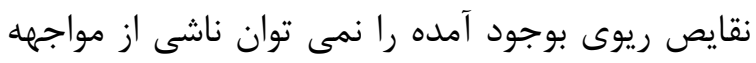

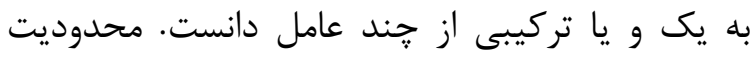

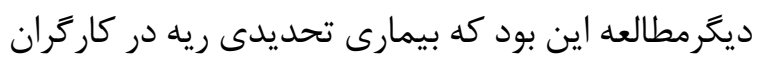

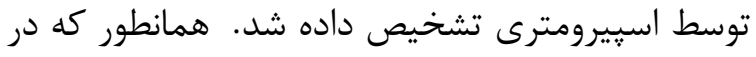

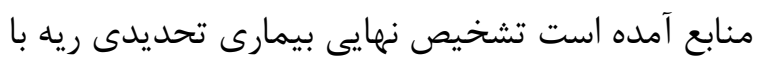

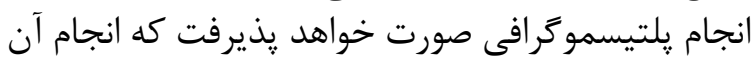
در اين مطالعه مقدور نبوده است.

$$
\text { تشكر و قدردانى }
$$

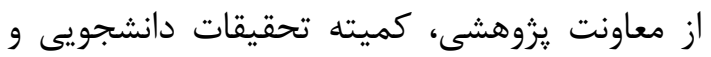

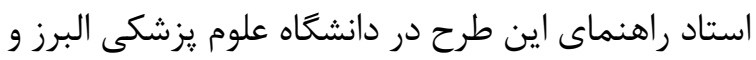

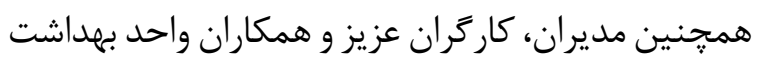

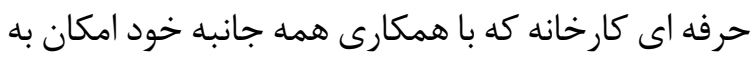

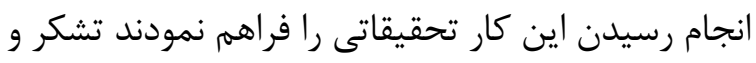

$$
\text { قدردانى مى تردد. }
$$

منابع

1. Beckett WS. Occupational respiratory diseases. New England Journal of Medicine. $2000 \mathrm{Feb}$ 10;342(6):406-13.

2. Rabe KF, Hurd S, Anzueto A, Barnes PJ, Buist SA, Calverley P, Fukuchi Y, Jenkins C, Rodriguez-Roisin R, Van Weel C, Zielinski J. Global strategy for the diagnosis, management, and prevention of chronic obstructive pulmonary disease: GOLD executive summary. American journal of respiratory and critical care medicine. $2007 \mathrm{Sep}$ 15;176(6):532-55.

3. Winder C, Stacey NH, editors. Occupational toxicology. CRC press; 2004 Feb 11.

4. Dalvie MA, White N, Raine R, Myers JE, London L, Thompson M, Christiani DC. Long-term respiratory health effects of the herbicide, 
22. Kullman GJ, Thorne PS, Waldron PF, Marx JJ, Ault B, Lewis DM, Siegel PD, Olenchock SA, Merchant JA. Organic dust exposures from work in dairy barns. American Industrial Hygiene Association Journal. 1998 Jun 1;59(6):403-13.

23. O'toole EA, Goel M, Woodley DT. Hydrogen peroxide inhibits human keratinocyte migration. Dermatologic surgery. 1996 Jun;22(6):525-9. erythrocytes prevent hydrogen peroxide-mediated damage to isolated perfused rat lungs and cultured bovine pulmonary artery endothelial cells. The Journal of clinical investigation. 1984 Jul 1;74(1):292-5.

21. Hoyt KR, Gallagher AJ, Hastings TG, Reynolds IJ. Characterization of hydrogen peroxide toxicity in cultured rat forebrain neurons. Neurochemical research. 1997 Mar;22(3):333-40. 